\title{
SISTEMAS DE COLHEITA DA CANA-DE-AÇÚCAR E ALTERAÇÕES NAS PROPRIEDADES FíSICAS DE UM SOLO PODZÓLICO AMARELO NO ESTADO DO ESPÍRITO SANTO'
}

\author{
MARCOS BACIS CEDDIA ${ }^{2}$, LÚCIA HELENA CUNHA DOS ANJOS ${ }^{3}$, EDUARDO LIMA ${ }^{3}$, \\ ALEXANDRE RAVELLI NETO ${ }^{3}$ e LUIZ ANTONIO DA SILVA ${ }^{4}$
}

\begin{abstract}
RESUMO - Foi instalado um experimento de longa duração (1989-1994) com cana-de-açúcar (variedade RB 739735) no município de Linhares, ES, com o objetivo de avaliar o efeito dos seguintes sistemas de colheita da cana-de-açúcar: a) Sistema Cana Crua - corte da cana sem queima, com posterior espalhamento do palhiço sobre o solo; e b) Sistema Cana Queimada - corte da cana com queima prévia do palhiço, sobre algumas propriedades físicas de solo Podzólico Amarelo em área de tabuleiro. O delineamento utilizado foi o de blocos casualizados, com seis repetições. Após seis anos de cultivo, constatou-se alteração do solo no sistema Cana Queimada, evidenciada pela diminuição do diâmetro médio ponderado dos agregados estáveis em água e pelo aumento da densidade do solo na profundidade de $0-5 \mathrm{~cm}$. Foram também detectadas alterações significativas na porosidade total e distribuição de poros, na profundidade de $0-5 \mathrm{~cm}$, devido às práticas de manejo do sistema de colheita. Verificou-se, ainda, que a velocidade de infiltração instantânea foi maior nas áreas sob tratamento sem queima. Os mesmos resultados não foram encontrados quando se avaliou o fluxo de água saturado através do método do permeâmetro.
\end{abstract}

Termos para indexação: estabilidade de agregados, densidade do solo, Saccharum officinarum, manejo de colheita, palhiço.

\section{SUGAR CANE HARVESTING SYSTEMS AND CHANGES ON PHYSICAL PROPERTIES OF A YELLOW PODZOLIC SOIL IN ESPÍRITO SANTO, BRAZIL}

\begin{abstract}
A long term (1989-1994) experiment with sugar cane (RB 739735) was carried out, in Linhares, ES, Brazil, in order to evaluate the effect of two systems of sugar cane harvesting: the first with previous burning (burned cane), and the second with crop residue remaining on the soil surface (green cane), on some physical properties of a tableland Yellow Podzolic soil. The statistical design was in randomized blocks, replicated six times. After six years of cultivation, a process of soil degradation was observed in the burned cane system, with decreasing of aggregate mean diameter and increasing of soil bulk density at the $0-5 \mathrm{~cm}$ soil depth. There were also differences in total porosity and porous distribution at the $0-5 \mathrm{~cm}$ soil depth as a consequence of the harvesting system. The soil water infiltration, measured in the field, was greatest in the area under the green cane treatments. The same result was not observed when measuring saturated hydraulic conductivity with the steady state permeameter.
\end{abstract}

Index terms: stability of aggregates, soil density, Saccharum officinarum, harvesting management, crop residue.

${ }^{1}$ Aceito para publicação em 19 de novembro de 1998. Extraído da Dissertação de Mestrado apresentada pelo primeiro autor à UFRRJ.

${ }^{2}$ Eng. Agr., M.Sc., Prof. Assistente, Dep. de Solos, Univ. Fed. Rural do Rio de Janeiro (UFRRJ), BR-465, CEP 23890-000 Seropédica, RJ. E-mail: ceddia@ufrrj.br

${ }^{3}$ Eng. Agr., Ph.D., Prof. Adjunto, Dep. de Solos, UfRRJ.

${ }^{4}$ Eng. Agr., CAPES/CPGCS/UFRRJ.

\section{INTRODUÇÃO}

As atuais técnicas de manejo da cultura da cana-de-açúcar (Saccharum officinarum L.) utilizam um vigoroso revolvimento por ocasião do plantio, com o uso de arados, grades pesadas e subsoladores. Ainda, ao fim do ciclo de produção, é comumente realizada a queima da palhada da cana antes da 
colheita, o que é prejudicial à manutenção dos níveis de matéria orgânica do solo. $\mathrm{O}$ aporte de matéria orgânica bruta é reduzido, e a prática da queima favorece a mineralização da matéria orgânica já existente. Além disso, o solo permanece descoberto por um período relativamente longo, o que acelera o processo erosivo e culmina com a depauperação de suas propriedades físicas e químicas (Molina, 1995; Mendoza, 1996).

Os solos de tabuleiro na região de Campos dos Goytacazes, RJ, após 20 ou 30 anos de cultivos consecutivos com cana-de-açúcar, apresentam produtividades médias da ordem de 40 toneladas de colmos por hectare, contra uma média de 80 toneladas por hectare em outras áreas de tabuleiro menos degradadas (Azeredo, 1994). No Estado do Espírito Santo, muitas das áreas recém-incorporadas ao processo produtivo são de solos de tabuleiros, que até então não estavam sendo utilizados ou de uso restrito a pastagens e florestas. Essas áreas apresentam produtividade de cerca de 75 toneladas por hectare na média de quatro cortes. Porém, continuando o atual sistema de manejo, como já observado em outras regiões, as produtividades deverão ser cada vez menores e dependerão do aumento do uso de insumos, como fertilizantes e corretivos.

Vários estudos de manejo de solo têm demonstrado a importância do fornecimento de matéria orgânica visando à manutenção e melhoria das propriedades físicas do solo (Gomes et al., 1978; Oliveira et al., 1983; Oades, 1984; Cerri et al., 1991). Existem várias possibilidades técnicas para manter ou aumentar os níveis de matéria orgânica nos solos de tabuleiro. Entre as práticas, a de manejo mais simples e que pode apresentar resultados técnicos e econômicos a curto e médio prazo é a do corte da cana sem queimar as folhas, deixando-as sobre o solo.

O objetivo deste trabalho foi avaliar o efeito de dois sistemas de manejo do palhiço da cana-de-açúcar antes da colheita, sobre algumas propriedades físicas de um solo Podzólico Amarelo em área de tabuleiro no Estado do Espírito Santo.

\section{MATERIAL E MÉTODOS}

Um experimento de longa duração foi instalado em 28 de maio de 1989, em solo classificado como Podzólico Amarelo distrófico, horizonte A moderado, e em área pertencente à LAGRISA (Linhares Agrícola S/A), no municí- pio de Linhares (paralelos $19^{\circ} 06^{\prime}$ e $19^{\circ} 18^{\prime}$ latitude sul e meridianos $39^{\circ} 43^{\prime}$ e $40^{\circ} 19^{\prime}$ longitude oeste $\mathrm{GW}$ e altitude de 30 metros), incluída na zona fisiográfica denominada Baixo Rio Doce, no Estado do Espírito Santo (Embrapa, 1978). Os tratamentos avaliados foram os sistemas de manejo de colheita da cana-de-açúcar com: a) corte da cana sem queima do palhiço (folhas secas mais pontas, material não-triturado) e com distribuição deste na superfície do solo, (Cana Crua), e b) corte da cana após queima do palhiço (Cana Queimada). O delineamento experimental foi o de blocos casualizados. A área experimental, que antes encontrava-se com pastagem, foi constituída de seis blocos, distando $2 \mathrm{~m}$ entre si. Cada bloco tinha 28,4 metros de largura e 95 metros de comprimento (2.698 $\mathrm{m}^{2}$ de área), com onze linhas de cana em cada tratamento (espaçamento de 1,2 m entre linhas). Por ocasião da colheita, foram excluídas as linhas $1,2,10 \mathrm{e}$ 11, referentes à bordadura, resultando em área útil de $798 \mathrm{~m}^{2}$ por tratamento. A variedade utilizada foi a RB 739735, que apresenta porte ereto e fácil despalha. No plantio, foram aplicados no sulco $125 \mathrm{~kg}$ de $\mathrm{P}_{2} \mathrm{O}_{5}$, $85 \mathrm{~kg}$ de $\mathrm{K}_{2} \mathrm{O}$ e $500 \mathrm{~kg}$ de calcário dolomítico por hectare. Nos anos seguintes (1990-1994), após as colheitas, fez-se a adubação das soqueiras com $400 \mathrm{~kg}$ de 25-00-20 por hectare.

Em 1994, após cinco anos de cultivo, foram realizadas amostragens para determinação dos seguintes parâmetros físicos: densidade do solo e das partículas, granulometria, grau de floculação, estabilidade de agregados, porosidade total e distribuição de poros.

Amostras indeformadas foram retiradas com o coletor Uhland nas profundidades de $0-5$ e $5-10 \mathrm{~cm}$ (coletor Uhland adaptado com anéis de $5 \mathrm{~cm}$ de altura), e de 10-20, 20-30 e 30-40 cm (coletor Uhland de 7,5 cm de altura), em minitrincheiras. Três locais de amostragem foram dispostos dentro de cada parcela, distanciados de 10,20 e 30 metros da cabeceira das parcelas, entre as linhas 3 e 4, 6 e 7 e 9 e 10. Nas amostras obtidas com o coletor de Uhland determinou-se a condutividade hidráulica saturada, pelo método do permeâmetro de carga constante (Embrapa, 1979). Após a determinação da condutividade hidráulica, procedeu-se, nas mesmas amostras, às análises de porosidade total e distribuição de poros pelo método da mesa de tensão (Embrapa, 1979). Finalmente, as amostras foram colocadas em estufa a $110^{\circ} \mathrm{C}$, e após um dia de secagem obteve-se a massa do solo seco, determinando-se assim a densidade do solo.

As amostras para a análise de estabilidade de agregados foram coletadas nas mesmas profundidades das amostras para determinação de densidades e porosidade do solo, porém com auxílio de pá reta. O método de análise 
utilizado foi o de Yoder (1936). As amostras foram também usadas para a análise granulométrica, pelo método da pipeta (Embrapa, 1979).

Para as determinações de velocidade de infiltração instantânea da água no solo foram construídos "diques" nas entrelinhas da cultura, de $1 \mathrm{~m}^{2}$ de área e $15 \mathrm{~cm}$ de altura (Reichardt, 1987). Nesse método não se objetivou caracterizar a condutividade hidráulica saturada $\left(\mathrm{K}_{\mathrm{qs}}\right)$ do solo, e sim, comparar tratamentos. Desta forma, no interior de cada dique, colocou-se uma lâmina de $100 \mathrm{~mm}$, e marcou-se o tempo necessário para a infiltração desse volume de água. Foram feitos seis testes por bloco (três por tratamento) a 10, 20 e 30 metros da cabeceira de cada parcela, totalizando 36 testes. Foi registrada a precipitação pluvial média em Linhares (Fig. 1) e determinada a umidade do solo quando da realização dos testes de infiltração (Fig. 2).

\section{RESULTADOS E DISCUSSÃO}

$\mathrm{Na}$ determinação dos fatores que podem influenciar as propriedades físicas do solo de tabuleiro nos tratamentos avaliados, é necessário caracterizar as diferenças entre os sistemas de manejo da colheita de cana-de-açúcar. No tratamento Cana Queimada, a quantidade de material vegetal deixado na superfície do solo é relativamente menor, por um período

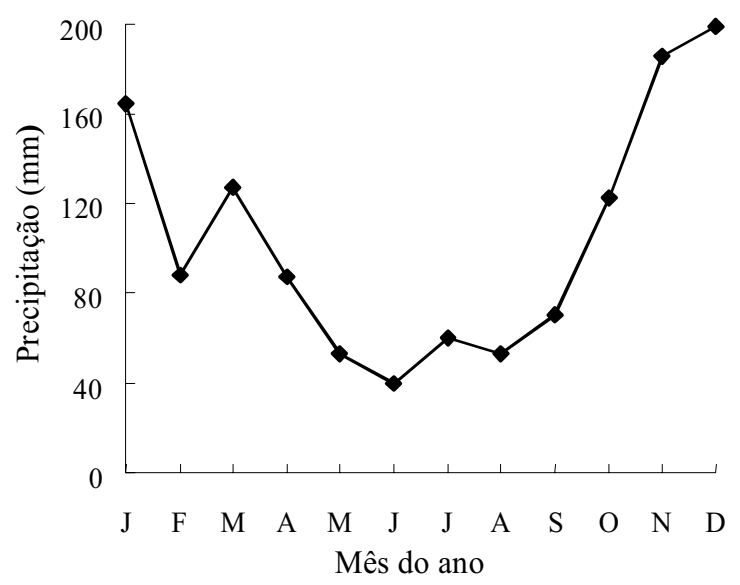

FIG. 1. Variação média da pluviosidade no município de Linhares, ES, no período de 1970-1995 (Fonte: Sexto distrito de meteorologia, EMCAPA/Linhares, ES). significativo da cultura da cana-de-açúcar (3-4 meses iniciais, entre a colheita e o início do fechamento das entrelinhas). Por outro lado, no tratamento Cana Crua foi adicionada maior massa de resíduos orgânicos (Tabela 1), o que permite maior interação entre as frações orgânica e mineral do solo, bem como a proteção da superfície do terreno contra o impacto de gotas de chuva e da água de irrigação.

A adição de matéria orgânica fresca na forma de palhada (folhas e pontas) foi, em média, de 16,7 toneladas por hectare (Tabela 1) no tratamento Cana Crua. Esse material em decomposição origina uma série de compostos orgânicos, tais como: açúcares, aminoácidos, ceras, fenóis, ligninas e ácidos (Shinitzer, 1991), que promovem a ligação entre as partículas e/ou reduzem a molhabilidade da superfície do agregado (Emerson et al., 1986). Mendoza (1996), em trabalho realizado com fracionamento da matéria orgânica, constatou que a manutenção da palhada na superfície do solo, no tratamento Cana Crua, proporcionou aumento significativo no teor de humina e carbono da fração ácidos fúlvicos.

O diâmetro médio ponderado (DMP) dos agregados estáveis apresenta padrão de comportamento diferente entre os tratamentos (Tabela 2). No tratamento Cana Queimada, verificou-se aumento significativo e proporcional do DMP com a

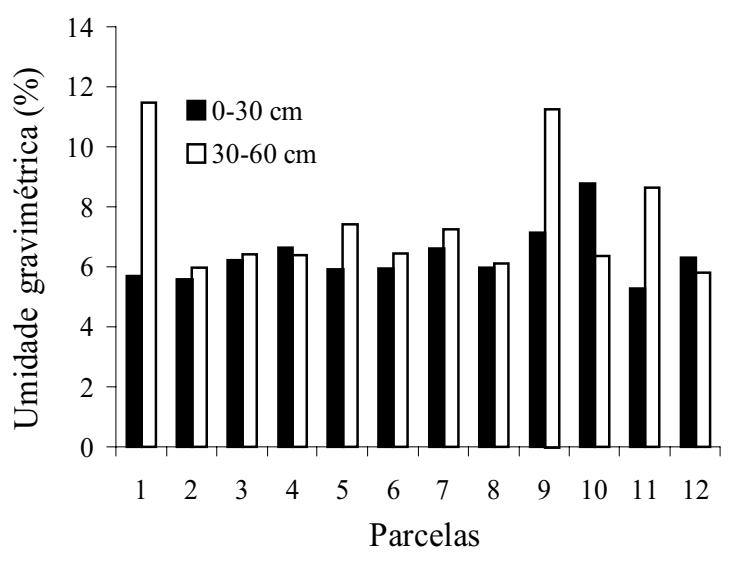

FIG. 2. Umidade do solo durante os testes de infiltração, no ano de 1994.

Pesq. agropec. bras., Brasília, v.34, n.8, p.1467-1473, ago. 1999 
profundidade, enquanto no tratamento Cana Crua os diâmetros tenderam a diminuir com a profundidade. Quando analisadas as diferenças para cada profundidade de solo, observa-se que: a) na camada de 0-5 cm, o DMP é maior no sistema Cana Crua, devido ao efeito do manejo, e b) a partir de $20 \mathrm{~cm}$ de profundidade ocorre uma inversão, com maiores valores de DMP no sistema Cana Queimada, não explicável por diferenças nos sistemas de colheita.
O DMP dos agregados (Tabela 2) é significativamente menor na profundidade de $0-5 \mathrm{~cm}$ no sistema de manejo Cana Queimada, o que demonstra que, com o tempo, nesse sistema, ocorre um processo de destruição dos agregados do solo. A individualização das partículas, antes componentes do agregado, aumenta a densidade do solo, conforme verificado pela análise dos dados na Tabela 2. A conservação dos agregados no tratamento Cana Crua foi também

TABELA 1. Produção (t ha-1) de colmos, folhas e peso total da cana-planta e socas sucessivas, no período de 1989-1994. Média de seis repetições ${ }^{1}$.

\begin{tabular}{|c|c|c|c|c|c|c|c|c|c|c|c|c|}
\hline \multirow[t]{2}{*}{ Material } & \multicolumn{2}{|c|}{ Cana-planta } & \multicolumn{2}{|c|}{ Primeira soca } & \multicolumn{2}{|c|}{ Segunda soca } & \multicolumn{2}{|c|}{ Terceira soca } & \multicolumn{2}{|c|}{ Quarta soca } & \multicolumn{2}{|c|}{ Quinta soca } \\
\hline & $\mathrm{CC}$ & CQ & $\mathrm{CC}$ & CQ & $\mathrm{CC}$ & CQ & $\mathrm{CC}$ & CQ & $\mathrm{CC}$ & CQ & $\mathrm{CC}$ & CQ \\
\hline Colmos & 138,0 & 146,2 & 91,2 & 96,0 & 73,1 & 66,6 & 51,6 & 63,7 & 46,0 & 47,5 & 71,9 & 65,6 \\
\hline $\begin{array}{l}\text { Folhas e } \\
\text { pontas }\end{array}$ & 17,2 & 17,9 & 14,9 & 16,1 & 15,3 & 15,2 & 13,6 & 17,9 & 12,6 & 13,3 & 26,6 & 27,1 \\
\hline Total & 155,2 & 164,1 & 106,1 & 112,1 & 88,4 & 81,8 & 65,2 & 81,6 & 58,6 & 60,8 & 98,5 & 92,7 \\
\hline
\end{tabular}

${ }^{1}$ CC: cana crua; CQ: cana queimada.

TABELA 2. Propriedades físicas do solo em diferentes profundidades nos sistemas cana crua e cana queimada. Média de seis repetições ${ }^{1}$.

\begin{tabular}{|c|c|c|c|c|c|c|}
\hline Tratamento & Profundidade & Poros & Macroporos & Microporos & Ds & $\mathrm{DMP}^{2}$ \\
\hline \multirow{6}{*}{ Cana crua } & $(\mathrm{cm})$ & ---------. & --- $(\%)---$ & -------------- & $\left(\mathrm{kg} \mathrm{dm}^{-3}\right)$ & $(\mathrm{mm})$ \\
\hline & $0-5$ & $42 \mathrm{Aa}$ & $25 \mathrm{Aa}$ & 17Aab & $1,50 \mathrm{Bb}$ & $4,5 \mathrm{Aa}$ \\
\hline & $5-10$ & 39Aa & $23 \mathrm{Aa}$ & $15 \mathrm{Ab}$ & 1,59Aa & $4,1 \mathrm{Aa}$ \\
\hline & $10-20$ & $38 \mathrm{Aa}$ & $21 \mathrm{Aab}$ & $17 \mathrm{Aab}$ & $1,56 \mathrm{Aab}$ & $4,1 \mathrm{Aa}$ \\
\hline & $20-30$ & $41 \mathrm{Aa}$ & $23 \mathrm{Aab}$ & $18 \mathrm{Aab}$ & $1,55 \mathrm{Aab}$ & $3,8 \mathrm{Aa}$ \\
\hline & $30-40$ & $40 \mathrm{Aa}$ & $19 \mathrm{Ab}$ & $21 \mathrm{Aa}$ & $1,50 \mathrm{Ab}$ & $3,8 \mathrm{Aa}$ \\
\hline \multirow[t]{5}{*}{ Cana queimada } & $0-5$ & $38,0 \mathrm{Ba}$ & $25 \mathrm{Aa}$ & $13 \mathrm{Bc}$ & 1,59Aa & $3,5 \mathrm{Bb}$ \\
\hline & $5-10$ & $38,0 \mathrm{Aa}$ & $23 \mathrm{Aab}$ & $15 \mathrm{Abc}$ & $1,60 \mathrm{Aa}$ & $3,6 \mathrm{Aab}$ \\
\hline & $10-20$ & $38,0 \mathrm{Aa}$ & $19 \mathrm{Ac}$ & $19 \mathrm{Aab}$ & $1,57 \mathrm{Aa}$ & $4,0 \mathrm{Aab}$ \\
\hline & $20-30$ & $41,5 \mathrm{Aa}$ & $20 \mathrm{Abc}$ & $21 \mathrm{Aa}$ & $1,52 \mathrm{Aab}$ & $4,1 \mathrm{Aab}$ \\
\hline & $30-40$ & $41,5 \mathrm{Aa}$ & $18 \mathrm{Ac}$ & $23 \mathrm{Aa}$ & $1,46 \mathrm{Bb}$ & $4,2 \mathrm{Aa}$ \\
\hline $\mathrm{CV}(\%)$ & - & 5,6 & 12,6 & 16,4 & 3,1 & 10,3 \\
\hline
\end{tabular}

${ }^{1}$ Médias seguidas de letras maiúsculas iguais não diferem significativamente a 5\%, pelo teste t de Student, entre tratamentos em cada profundidade; médias seguidas de letras minúsculas iguais não diferem significativamente a $1 \%$, pelo teste de Tukey, entre profundidades.

2 Diâmetro médio ponderado dos agregados estáveis. 
observada por Baxter (1983), o que evidencia que o sistema de corte sem queima da palha possibilita a melhoria ou manutenção dos agregados.

Os valores de densidade do solo (Tabela 2), em geral, foram superiores a $1,50 \mathrm{~kg} \mathrm{dm}^{-3}$, o que confirma as informações de Anjos (1985) e Fonseca (1986), que constataram densidades relativamente elevadas nos solos de tabuleiro.

O sistema de manejo Cana Queimada, na profundidade de $0-5 \mathrm{~cm}$, apresentou densidade do solo significativamente superior à do sistema de manejo Cana Crua (Tabela 2); isto confirma um processo de compactação superficial do solo. A causa da compactação das camadas superficiais está relacionada à diminuição do tamanho e quantidade dos agregados do solo. Segundo Cassel \& Lal (1992), a perda de matéria orgânica, em áreas recentemente exploradas, favorece a compactação, sendo, em solos tropicais, a maior causa desse processo o tipo de manejo das terras. Os resultados obtidos concordam com os de Gomes et al. (1978), que estudaram o impacto de sistemas de manejo e tempo de cultivo em solo Podzólico Vermelho-Amarelo, e de Anjos et al. (1994) que avaliaram as variações nas propriedades físicas de solos sob diferentes sistemas de uso e manejo.

O fato de a superfície do solo no sistema Cana Queimada ficar exposta aos impactos de gotas de chuva (principalmente no período pós-colheita, que coincide com as maiores taxas de precipitações pluviais mensais), também influi no aumento da densidade do solo. A compactação do solo, causada por esse fator, manifesta-se mais facilmente em solos com fraca agregação, baixo conteúdo de matéria orgânica e altas porcentagens de silte (Cassel \& Lal, 1992).

$\mathrm{Na}$ profundidade de 30-40 cm também se constatou que a densidade, na área com tratamento Cana Crua, foi significativamente maior, mas essas alterações se devem, provavelmente, à variabilidade ao longo do perfil do solo.

A porosidade total do solo diminuiu de forma significativa no tratamento Cana Queimada, na profundidade de $0-5 \mathrm{~cm}$ (Tabela 2), o que é coerente com os dados de estabilidade de agregados e densidade do solo. No entanto, ocorreu diminuição significativa no percentual de microporos $(0-5 \mathrm{~cm})$.
Esses resultados diferem dos obtidos por Fernandes et al. (1984), que associaram a diminuição do diâmetro médio dos agregados à diminuição dos macroporos. É possível que dificuldades operacionais durante a coleta da amostra e determinação no laboratório (freqüentes em solos de textura arenosa), tenham causado modificações no arranjo do espaço poroso, impossibilitando a constatação de alterações decorrentes do manejo.

De conformidade com os valores de densidade do solo, na profundidade de $30-40 \mathrm{~cm}$, a porosidade total é significativamente menor no tratamento Cana Crua, comportamento este não explicável pelos sistemas de manejo.

Em relação à profundidade, a variação do volume de poros acompanhou a textura do solo (Tabela 3). Apesar de a porosidade total não se alterar com a profundidade, nas camadas superiores onde a textura é mais arenosa (cerca de 90\% de areia) ocorre o predomínio de macroporos, aproximadamente $60 \%$, contra $40 \%$ de microporos em relação à porosidade total. A partir de $30 \mathrm{~cm}$, onde há aumento do teor de argila (10 para 17\%), a microporosidade passa a representar cerca de $55 \%$ da porosidade total (Tabela 2).

$\mathrm{Na}$ Tabela 4 são apresentados os valores de fluxo de água no solo, obtidos pelos métodos do condutivímetro de carga constante, com a determinação da condutividade hidráulica saturada, e da construção de diques, com determinação da velocidade de infiltração instantânea. A condutividade hidráulica saturada não foi alterada significativamente pelo sistema de manejo da colheita da cana. Em profundidade, observa-se uma pequena redução do

TABELA 3. Análise granulométrica nas diferentes profundidades do solo. Média de 12 parcelas.

\begin{tabular}{ccccc}
\hline Profundidade & Areia & \multicolumn{2}{c}{ Silte Argila total Argila natural } \\
\hline$(\mathrm{cm})$ & $--------------(\%)$ & 8 & 4 \\
$0-5$ & 88 & 4 & 8 & 4 \\
$5-10$ & 89 & 3 & 8 & 4 \\
$10-20$ & 89 & 3 & 8 & 6 \\
$20-30$ & 85 & 5 & 10 & 11 \\
$30-40$ & 78 & 5 & 17 & \\
\hline
\end{tabular}


TABELA4. Fluxo de água no solo $\left(\mathrm{m}^{3} \mathrm{~m}^{-2} \mathrm{~s}^{-1} \times 10^{-3}\right)$. Média de seis repetições.

\begin{tabular}{|c|c|c|c|}
\hline \multirow[t]{2}{*}{ Tratamento } & \multirow{2}{*}{$\begin{array}{l}\text { Profundidade } \\
\text { (cm) }\end{array}$} & \multicolumn{2}{|c|}{ Fluxo de água no solo } \\
\hline & & \multicolumn{2}{|c|}{$\begin{array}{l}\text { Condutividade Infiltração } \\
\text { hidraúlica }\end{array}$} \\
\hline \multirow[t]{5}{*}{ Cana crua } & $0-5$ & $1,07^{\mathrm{ns}}$ & \\
\hline & $5-10$ & $1,01^{\mathrm{ns}}$ & \\
\hline & $10-20$ & $1,09^{\mathrm{ns}}$ & $0,56 a$ \\
\hline & $20-30$ & $1,33^{\mathrm{ns}}$ & \\
\hline & $30-40$ & $0,97^{\mathrm{ns}}$ & \\
\hline \multirow[t]{5}{*}{ Cana queimada } & $0-5$ & $1,23^{\mathrm{ns}}$ & \\
\hline & $5-10$ & $1,24^{\mathrm{ns}}$ & \\
\hline & $10-20$ & $1,01^{\mathrm{ns}}$ & $0,38 b$ \\
\hline & $20-30$ & $1,06^{\mathrm{ns}}$ & \\
\hline & $30-40$ & $0,96^{\mathrm{ns}}$ & \\
\hline CV (\%) & - & 32,1 & 13,4 \\
\hline
\end{tabular}

fluxo de água, mas essa redução é, provavelmente, resultante da mudança na textura.

A velocidade de infiltração instantânea da água no solo (Tabela 4), ao contrário da condutividade hidráulica saturada, foi significativamente menor no tratamento Cana Queimada. Tal resultado está de acordo com as variações na estabilidade dos agregados estáveis em água e na densidade do solo (Tabela 2). Provavelmente, a compactação da camada de $0-5 \mathrm{~cm}$ do solo determinou o impedimento à infiltração da água no solo, evidenciado de forma mais eficiente pelo método de construção de diques na superfície do solo.

\section{CONCLUSÕES}

1. No sistema Cana Queimada, a degradação das propriedades do solo é evidenciada pela redução do diâmetro médio ponderado dos agregados estáveis e pelo aumento da densidade do solo na profundidade de $0-5 \mathrm{~cm}$, com conseqüente diminuição da velocidade de infiltração instantânea da água no solo.

2. O método de determinação do fluxo de água pela construção de diques é mais eficiente na avaliação do efeito do manejo da colheita da cana-de-açúcar na dinâmica da água em solo Podzólico Amarelo de tabuleiro.

\section{AGRADECIMENTOS}

À LAGRISA (Linhares Agrícola S/A), pelo apoio de campo; às agências de fomento CAPES/CNPq, pelo financiamento aos pesquisadores; e aos técnicos e pesquisadores da Estação Experimental Dr. Leonel Miranda/UFRRJ, pelo apoio ao projeto de pesquisa.

\section{REFERÊNCIAS}

ANJOS, J.T.; UBERTI, A.A.A.; VIZZOTO, V.G.; LEITE, G.B.; KRIEGER, M. Propriedades físicas em solos sob diferentes sistemas de uso e manejo. Revista Brasileira de Ciência do Solo, Campinas, v.18, n.1, p.139-145, 1994.

ANJOS, L.H.C. dos. Caracterização, gênese, classificação e aptidão agrícola de uma seqüência de solos do Terciário na região de Campos, RJ. Itaguaí: UFRRJ, 1985. 194p. Dissertação de Mestrado.

AZEREDO, D.F. Colheita da cana-de-açúcar com queima e sem queima. Campos dos Goytacazes: UFRRJ, Estação Experimental Dr. Leonel Miranda, 1994. 46p. (Boletim técnico/ sem número).

BAXTER, B. Green cane harvest review (the merits of blanketing). Australian Canegrower, Melbourne, v.5, n.5, p.95-97, 1983.

CASSEL, D.K.; LAL, R. Soil physical properties of the tropics: Common beliefs and management restraints. In: LAL, R.; SANCHEZ, P.A. (Eds.). Myths and science of soils of the tropics. Madison: Soil Science Society of America, 1992. p.61-89 (Special Publication, n.29).

CERRI, C.C.; FELLER, C.; CHAUVEL, A. Evolução das principais propriedades de um Latossolo Vermelho-Escuro após desmatamento e cultivo por doze e cinqüenta anos com cana-de-açúcar. Cahiers Orstom, Série Pédologie, Bondy, v.26, p.37-50, 1991.

EMBRAPA. Serviço Nacional de Levantamento e Conservação de Solos (Rio de Janeiro, RJ). Levantamento de reconhecimento dos solos do Estado do Espírito Santo. Rio de Janeiro, 1978. 161p. (Boletim técnico, 45). 
EMBRAPA. Serviço Nacional de Levantamento e Conservação dos Solos (Rio de Janeiro, RJ). Manual de métodos de análise de solos. Rio de Janeiro, 1979. v.1.

EMERSON, W.W.; FOSTER, R.C.; OADES, J.M. Organo-mineral complexes in relation to soil aggregation and structure. In: HUANG, P.M.; SCHNITZER, M. (Eds.). Interactions of soil minerals with natural organics and microbes. Madison: Soil Science Society of America, 1986. p.521-548. (Special Publication, n.17).

FERNANDES, J.; RIPOLI, T.C.; MILLAN, M. A compactação do solo e a brotação das soqueiras. Informativo Álcool e Açúcar, v.34, p.12-17, 1984.

FONSECA, O.O.M. da. Caracterização e classificação de solos Latossólicos e Podzólicos desenvolvidos nos sedimentos do Terciário no litoral brasileiro. Itaguaí: UFRRJ, 1986. 185p. Dissertação de Mestrado.

GOMES, A.S.; PATELlA, J.F.; PAUletTO, E.A. Efeito de sistemas e tempo de cultivo sobre a estrutura de um solo Podzólico Vermelho-Amarelo textura média/argilosa. Revista Brasileira de Ciência do Solo, Campinas, v.2, n.1, p.17-21, 1978.

MENDOZA, H.N.S. Efeitos de sistemas de colheita dos canaviais sobre propriedades químicas e biológicas em solo de tabuleiro no Espírito Santo. Seropédica: UFRRJ, 1996. 112p. Dissertação de Mestrado.

MOLINA, R.M. A torta de filtro e o bagaço no comportamento da biota, propriedades físicas e produtividade de um solo cultivado em cana-de-açúcar. Seropédica: UFRRJ, 1995. 96p. Tese de Doutorado.

OADES, J.M. Soil organic matter and structural stability: Mechanisms and implications for management. Plant Soil, Netherlands, v.76, p.319-337, 1984.

OLIVEIRA, M.; CURI, N.; FREIRE, J.C. Influência do cultivo na agregação de um Podzólico Vermelho-Amarelo textura média/argilosa (MG). Revista Brasileira de Ciência do Solo, Campinas, v.7, p.317-322, 1983.

REICHARDT, K. A água em sistemas agrícolas. São Paulo: Manole, 1987. 188p.

SHINITZER, M. Soil organic matter. The next 75 years. Soil Science, Baltimore, v.151, n.1, p.41-58, 1991.

YODER, R.E. A direct method of aggregate analysis of soils and a study of the physical nature of erosion losses. Journal of American Society of Agronomy, Madison, v.28, p.337-351, 1936. 